\title{
A massive and dense core in an early stage of evolution
}

\section{Yanett Contreras ${ }^{* \dagger}$}

Universidad de Chile, Max-Planck-Institut fãijr Radioastronomie

E-mail: yanettedas.uchile.cl

\section{Guido Garay}

Universidad de Chile

E-mail: guido@das.uchile.cl

\section{Diego Mardones}

Universidad de Chile

E-mail:diego@das.uchile.cl

\begin{abstract}
We present molecular line observations towards the cold core G305.136+0068 in the $\mathrm{CO}(3-2)$, 13CO(3-2) and CS(7-6) transitions, made with the ASTE telescope, and CS(2-1),CS(3-2) and CS(5-4) transitions made with the SEST telescope. We also present Spitzer/GLIMPSE images which show that the dust core is seen in silhouette in all the four IRAC bands and is surrounded by a shell of emission. We find three sources deeply embedded in this core, two of which are candidates for young high mass protostars still in the process of formation. Our observations support the hypothesis that G305.136+0068 is a massive and dense core in an early stage of evolution, in which the formation of high-mass stars have just started
\end{abstract}

From planets to dark energy: the modern radio universe

October 1-5 2007

University of Manchester, Manchester, UK

\footnotetext{
* Speaker.

${ }^{\dagger}$ Y.C., G.G. and D.M. acknowledge support from the Chilean Centro de Astrofisica (FONDAP 15010003)
} 


\section{Introduction}

G305.136+0.068 is a dust core with no MSX or IRAS counterparts, indicating it has a low dust temperature ( $<16 \mathrm{~K}$; Garay et al. 2004). We present here new observations made in order to investigate the physical conditions and kinematics of this cold core and its stellar content.

\section{Observations}

The observations were made using the Atacama Submillimeter Telescope (ASTE) and the Swedish-ESO Submillimeter telescope (SEST). The observed lines and parameters are given in Table 1.

\begin{tabular}{|c|c|c|c|c|c|}
\hline Line & Telescope & $\begin{array}{c}\text { Frequency } \\
\mathrm{MHz}\end{array}$ & $\begin{array}{c}\text { Beam } \\
\text { arcsec }\end{array}$ & Number of Maps & $\begin{array}{c}\text { Spacing } \\
\text { arcsec }\end{array}$ \\
\hline $\mathrm{CS}(2-1)$ & SEST & 97980.968 & 51.3 & 14 & 30 \\
\hline $\mathrm{CS}(3-2)$ & SEST & 146969.049 & 34.2 & 14 & 30 \\
\hline $\mathrm{CS}(5-4)$ & SEST & 244935.606 & 20.5 & 9 & 30 \\
\hline $\mathrm{CO}(3-2)$ & ASTE & 345795.990 & 21.8 & 25 & 30 \\
\hline${ }^{13} \mathrm{CO}(3-2)$ & ASTE & 330587.960 & 22.8 & 25 & 30 \\
\hline $\mathrm{CS}(7-6)$ & ASTE & 342882.950 & 22.0 & 9 & 30 \\
\hline
\end{tabular}

Table 1: Observed parameters

\section{Results}

- Emission was detected in all the observed molecular lines.

- The emission in all lines arises from nearly circularly symmetric structures. The size of the core is different in the different transitions, ranging from 0.15 to $0.5 \mathrm{pc}$ (assuming a distance of $3.4 \mathrm{kpc})$.

- The GLIMPSE images show that the dust core is seen in silhouette in all four IRAC bands and surrounded by a shell of emission (Fig. 1).

- We identified nine IRAC/SPITZER sources within a region of 20 " in radius centered at the peak of the dust core.

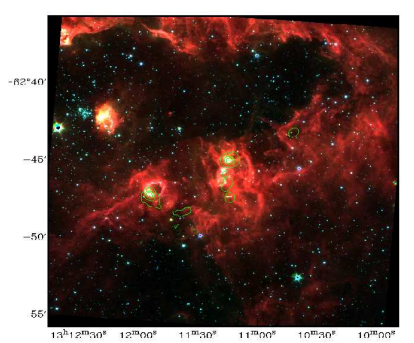

Figure 1: Spitzer image of the $3.6 \mu, 4.5 \mu$ and $8 \mu$ emission towards the $G 305.136+0.068$ region overlayed with the $50 \%$ contour level of the 1.2mm emission. The position of the cold core is indicated by the arrow. 


\section{Discussion}

\subsection{Core physical parameters}

From the $\mathrm{CO}(3 \rightarrow 2)$ and ${ }^{13} \mathrm{CO}(3 \rightarrow 2)$ observations we determined, assuming LTE excitation conditions, a core mass of $1.5 \times 10^{3} M_{\odot}$. For this estimation we adopted an excitation temperature of $15 \mathrm{~K}$ and a $\left[\mathrm{CO} /{ }^{13} \mathrm{CO}\right]$ abundance ratio of 50 .

From dust continuum observations at $1.2 \mathrm{~mm}$, Garay et al. (2004) estimated a mass of $1.1 \times 10^{3}$ $M_{\odot}$, assuming a dust temperature of $16 \mathrm{~K}$.

Another estimation can be obtained assuming that the core is in virial equilibrium. Using the values determined from the CS observations $\left(\Delta \mathrm{v}=6.0 \mathrm{~km} \mathrm{~s}^{-1}\right.$ and $\left.\mathrm{R}=0.15 \mathrm{pc}\right)$ we find a virial mass of $1.4 \times 10^{3} M_{\odot}$.

The masses and densities derived using the three different methods, summarized in Table 2, are in excellent agreement.

\begin{tabular}{ccc}
\hline Method & Mass $\left(M_{\odot}\right)$ & Density $\left(\mathrm{cm}^{-3}\right)$ \\
\hline LTE & $1.5 \times 10^{3}$ & $7.8 \times 10^{5}$ \\
Virial & $1.4 \times 10^{3}$ & $7.1 \times 10^{5}$ \\
Dust & $1.1 \times 10^{3}$ & $5.9 \times 10^{5}$ \\
\hline
\end{tabular}

Table 2: Derived parameters.

We conclude that the G305.136+0.068 dust core corresponds to a massive and dense core, with parameters similar to those of molecular cores harbouring already formed high-mass stars.

\subsection{Embedded Sources}

We found 9 IRAC/Spitzer sources within a radius of 20" centered at the peak position of the dust core. Five of these sources were detected in the 2MASS survey at near-infrared wavelengths. For the sources with enough data we fitted their SEDS using a large grid of precomputed models (Robitaille et al. 2007). We find that three IRAC sources are likely to be deeply embedded in the core, two of which are candidate for young high mass protostars still in the process of formation. The SEDs of these sources and model fits are presented in Fig. 2. The solid-line indicates the best fit, which in both cases corresponds to collapse models with large accretion rates of $\sim 1 \times$ $10^{-3} M_{\odot} y r^{-1}$.
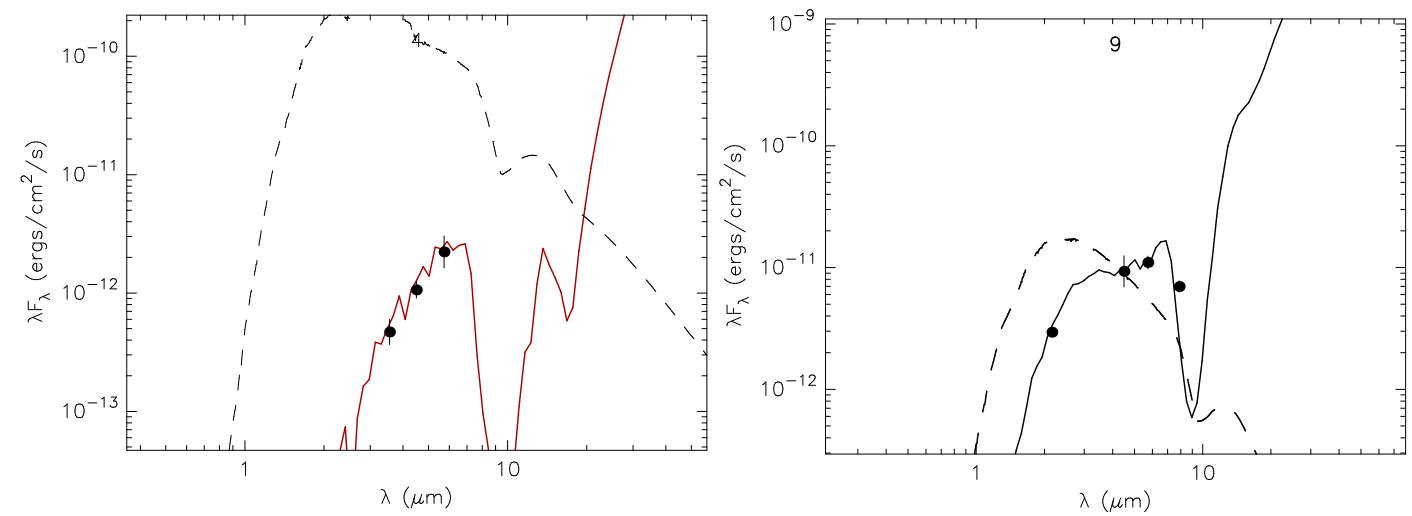

Figure 2:SEDs of embedded sources. Solid-line: best fit to the SED. Dotted-line: stellar photosphere model implied by the best fit model. 


\subsection{Modeling line spectra}

We are currently in the process of modeling the CS line profiles (in the four observed transitions) with model profiles obtained using a Monte Carlo radiative transfer code developed by Diego Mardones. The best model obtained so far, shown by the dotted line in Fig. 3, is a power law model with the density decreasing with radius as $n=1.4 \times 10^{5}\left(\frac{r}{0.1 p c}\right)^{-1.4}$ and the temperature decreasing inwards as $T=30\left(\frac{r}{0.1 p c}\right)^{0.7}$.

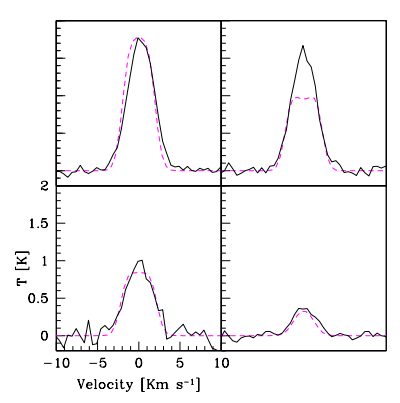

Figure 3: CS spectra observed at the peak position of the cold core. Dottedline: modeled spectra.

\section{Conclusions}

- The molecular line observations indicate that G305.136+0.068 is indeed a massive $(M \sim$ $\left.1.5 \times 10^{3} M_{\odot}\right)$ and very dense $\left(7.8 \times 10^{5} \mathrm{~cm}^{-3}\right)$ core. Molecular cores with these characteristics are thought to be the maternities of high-mass stars.

- The IRAC/SPITZER data indicates that the core harbors two high-mass YSOs undergoing accretion with high infall rates $\left(\sim 1 \times 10^{-3} M_{\odot} y r^{-1}\right)$.

- The new observations support the hypothesis that G305.136+0068 is a massive and dense core in an early stage of evolution, in which the formation of high-mass stars have just started.

\section{References}

[1] G. Garay et al., Discovery of four new dense and massive cold cores ,2004ApJ610,313G.

[2] T. Robitaille, SED modeling of Young Massive Stars, 2007arXiv0711.4369R 